\section{THE T.N.T. HEALTH HAZARD}

BY

\author{
HARRY M. ROBERTS, L.R.C.P.\&S.Ed., \\ L.R.F.P.S.Glasg. \\ Medical Officer, Nobel's Explosives Company, Ltd.
}

Whien toluol, or toluene, is completely nitrated with a mixed acid containing sulphuric and nitric acids, trinitrotoluene (T.N.T.) is produced. This is a pale yellow solid with a melting-point at about $80^{\circ} \mathrm{C}$. Nitration is completed at about $100^{\circ}$ C., the T.N.T. then separating out from the acid as a dark yellow oily liquid. The molten T.N.T. is washed with hot water until free of acid and is further washed with a solution of sulphite of soda to remove impurities. The purified T.N.T. is dried at $100^{\circ}$ to $110^{\circ} \mathrm{C}$. by running it through a steam-jacketed pan. The finished product can be obtained:

1. As flake, by cooling a film of molten T.N.T. on a revolving drum. A knife of brass which presses against the front of the drum removes the film as small flakes.

2. As crystalline powder, by cooling the molten T.N.T. in a pan, with slow continuous stirring. Lumps are removed by sieving.

3. As slab or biscuit, by allowing the molten T.N.T. to solidify in shallow pans.

4. As crushed flake, by passing flake between rollers and sieving to remove the larger particles.

Flake and crushed flake can be pressed into slabs. Crystalline powder and crushed flake are used in explosive mixtures - e.g., baratol, amatol, ammonal, and military ammonal with charcoal. Thus T.N.T. workers may be exposed to: (1) inhalation of fume from the molten variety ; (2) inhalation of T.N.T. dust into the nose, mouth, and throat, with subsequent ingestion; and (3) skin absorption from contact with any of the above forms. Two of the above may coexist, for the vapour from molten T.N.T. will deposit as a crystalline dust on any surface colder than $70^{\circ} \mathrm{C}$. Thus a worker thought to have fume contact only may, in addition, be exposed to ingestion. The effect of entry of T.N.T. into the system, the signs denoting entry, and the symptoms which may exist without signs, together with all measures necessary to prevent absorption, should be known to those responsible for the health of workers. The carrying out of such measures, including selection of those employed, should, under management, be controlled by a medical man or woman. Neither the State-registered nurse nor the experienced supervisor is sufficient to maintain adequate check on the hazard, although each is of great help if co-operating. Medical practitioners who lack industrial experience or who are unfamiliar with T.N.T. medical literature can be completely surprised, as is shown by the fact that recently several cases of T.N.T. toxic jaundice have not been recognized as such until within a day or two of death. Informative references recommended are:

1. "The Origin, Symptoms, Pathology, Treatment and Prophylaxis of Toxic Jaundice observed in Munition Workers"; a discussion by certain Sections of the Royal Society of Medicine (January, 1917).

2. "Researches into the Toxic Properties of Trinitrotoluene," by Benjamin Moore, D.Sc., F.R.S. (On behalf of the Medical Research Committee ; 1916).

3. "Trinitrotoluene Poisoning." Officially communicated by the Ministry of Munitions. (Lancet, 1916, 2, 1026.)

Prof. Moore's report is an account of an investigation into the properties and chemical reactions of T.N.T. during which conclusive experiments to determine the modes of entrance of the compound into the body were made. He describes the characteristic facies met with in many T.N.T. workers, and concludes with a description of Webster's test for the presence of T.N.T. in the urine. This report, although published in 1917 , can be accepted as being up to date.

The Lancet article embodies the Moore report, and is presented under the following headings: (1) digestive troubles, (2) blood changes, (3) channels of absorption, (4) differential diagnosis, (5) post-mortem appearances, (6) predisposition, (7) preventive measures and treatment. In an addendum Webster's test is described, with advice as to how the result should be interpreted. This article, in referring to cases of T.N.T. toxic jaundice in munition workers, states that $67 \%$ were caused by amatol and only $6 \%$ by ammonal, notwithstanding that ammonal contained the larger proportion of T.N.T. It suggests that the greater amount of ammonium nitrate in amatol was responsible, on account of its hygroscopic nature, for facilitating the passage of T.N.T. through the skin. Whatever justification existed in 1916 for regarding the compounds mentioned as standard in respect of their T.N.T. content, it would not apply to-day, for both amatol and ammonal vary considerably in the proportion of T.N.T. present. That wetness of hands caused by ammonium nitrate aids the entry of T.N.T. through the skin is debatable. T.N.T. is quite insoluble in ammonium nitrate, and absorption from solution would not arise. Some skins of fine texture may suffer breakdown of the epithelium from ammonium nitrate, which acts not unlike brine, and so the way be paved for absorption; but it should be kept in mind that the percentage of T.N.T. present is the factor which is the hazard and that the greater the contact with T.N.T. the greater is the health risk.

\section{Schedule for Control of the Hazard}

1. Selection of workers.

2. Record of physical condition on entry.

3. Record of sick absences.

4. Periodic examination for signs of T.N.T. absorption.

5. Record of T.N.T. stains in grades-for example, slight, gross, extensive - and also sites, such as hands, feet, underclothing (shirts and stockings), overalls.

6. Co-operation with the works chemist in carrying out Webster's test; this to be done always by the same person if possible.

7. An arrangement that foremen, chargemen, supervisors, or other responsible persons draw attention to workers looking off colour and to any complaining of fatigue or breathlessness.

8. Insistence on provision of protective clothing and control of dust by adequate ventilation; also insistence on washing the hands before all meals, and both hands and face before going home.

9. A reserve of alternative work for those that have to be "stood off."

\section{Selection of Workers}

Selection is justified, for a definite health hazard exists, and under present conditions there should be no difficulty in placing rejected candidates elsewhere. Further, those susceptible to T.N.T. may not be protected by even the most satisfactory working conditions and meticulous supervision, and it is well to know the type from which they may spring. Cardinal points for guidance are age, average good medical history, fair but not necessarily high standard of physical fitness, and evidence of attention or inattention to personal hygiene. These factors possess a varying standard, according to whether the process is the manufacture of T.N.T. or the subsequent handling of the product as in shell and grenade filling. Not infrequently fewer health casualties are met with during the manufacture in bulk of a toxic substance than occur in workers using it as a component of other manufacture or in assembly. On manufacturing plant only men are employed in this country; their numbers are out of all proportion to the thousands of young women newly engaged in shell, bomb, 
or grenade filling, and it is concerning these that recommendations as to selection apply.

Age is important, and 19 years should be the minimum for T.N.T. contact. Below this the incidence of susceptibility is high. In the war of 1914-18 persons aged 14, 15, and 16 years died from T.N.T. toxic jaundice after five or six weeks' work. Age in the upward direction is not significant, for middle-aged and elderly workers have been employed for many years in T.N.T. processes without suffering in health. In making selection avoid persons who show sweaty palms, for the orifices of the sweat glands will be open during work, thereby promoting skin absorption. Persons who sleep in underclothing worn at work add by this habit to the health risk. Anyone with a history of jaundice should be rejected. A yellow tinge in the sclerotic, often seen in those suffering from constipation, should call for rejection, because the colour alone will cause confusion at the periodic medical examination, while should constipation exist it will probably be habitual, which is a further objection. Long tapering finger-nails should be discouraged by supervisors, particularly when the finger-tip is not suited for this fashion, as explosive may lodge under the nail and be subsequently ingested. Nail-biting is for the same reason very objectionable, and evidence of this habit should be looked for, as it is resorted to whenever the hands are idle.

For women of 19 years body weight in indoor clothes of $120 \mathrm{lb}$. and upwards should be aimed at, and no female under $105 \mathrm{lb}$. should be exposed to T.N.T. contact. Such light-weight young women may be quite fit for effort, but, whatever the reason, experience has shown that it is in this class that T.N.T. sickness is apt to occur.

Recording Physical Condition.-Among other items age, height, weight, and history of anaemia or jaundice should not be missed. The weight will be of value later when dealing with cases under observation. Recent experience of many thousands of examinations is that in some areas a high percentage of women in the 20-30 year age group are $14 \mathrm{lb}$. underweight. A further point is that doctors are not furnished with physical details concerning persons allocated to them under the National Health Insurance Act, and when consulted by a new patient would not know if loss of weight had taken place.

Sick Absences.-For five months following entry into employment these should be recorded as a T.N.T. group and kept in a suspense file. At the termination of this probationary period all workers still fit can be regarded as normal in respect of exposure to T.N.T., but during the period absences certified as anaemia, gastritis, general debility, etc., should be the subject of investigation by the works medical officer, for often such certificates refer to a condition of malaise not definitely diagnosed. A Webster test at this stage is very useful, whether the result is negative or otherwise.

\section{Periodic Inspection for Signs of T.N.T. Absorption}

In the case of workers with less than four months' service inspection should be done weekly. After this period the frequency can be reduced, provided co-operation has been established between the medical officer and responsible persons on the job and with the welfare supervisor and factory nurse. A further essential to less frequent examination is that the medical officer should regularly scrutinize the entries recording attendance of workers at the ambulance room on account of indisposition or for such minor injuries as might facilitate the passage of T.N.T. through the skin. At the inspection the worker's hands should be examined and the T.N.T. facies looked for. On the hands brown tarry-looking stains may be found on the palms and the palmar aspect of the fingers. These emit an odour which can be detected if sought for, and impart a bitter taste to the tongue. Staining may be found on the soles of the feet if T.N.T. dust is spilt on the floors. Undue staining should be recorded, for the stain is a reservoir from which T.N.T. may continue to enter the system after the worker has been "taken off." Gross staining may point to carelessness, to failure to use protective clothing, and, if on the scalp, to absence of headcovering. There is evidence that T.N.T. may be slightly soluble in fats and oils, for a greaser on a T.N.T. plant will incur large intense stains more readily than other workers. The significance of staining depends on its extent. Most workers will show it in some degree, and in the absence of symptoms or of a marked facies (referred to later) no particular notice need be taken.

\section{The T.N.T. Facies}

This is characteristic, and consists of pallor comparable to that of shock, with coloration at the lips, lobes of the ears, and curl of the helix. This appearance has been referred to as cyanosis, and the lips have been described as " ashen grey." These terms, more or less correct, fail to convey an exact picture, for the hue is brighter than cyanosis-it is lilac in shade. On the lower lip it is so unreal as to convey the impression that a mistake has been made in the lipstick used. The sites mentioned are selective: the coloration is never seen on fingers or toes. After some experience the T.N.T. facies is easily recognized. Two important points should be mentioned: it is greatly modified by excitement or recent exercise on the part of the worker-for example, hurrying to be in time for the inspection-and surprisingly so by artificial light. Under illumination through mellow-tinted globes it disappears, but becomes obvious when the worker steps into the daylight.

The T.N.T. facies has been ascribed to oxygen starvation resulting from conversion to methaemoglobin (or nitricoxide-haemoglobin) of the oxyhaemoglobin of the red cell by T.N.T., and to a vasomotor phenomenon unrelated to any blood changes. Regarding the former, it is known that the facies may be present while the person concerned remains symptomless as regards lack of oxygen. He or she may carry on normally, unaware of alteration in wellbeing and showing no sign of fatigue or breathlessness. Further, the facies will exist when the red cell count and haemoglobin percentage are normal for the particular person. Oxygen has been liberally administered by me, without appreciable change, to persons showing the T.N.T. facies. It is a fact that when a worker with this facies is removed to another job he loses the colouring-a change credited to reoxygenation, but which may not be the correct explanation.

\section{Vasomotor Theory}

In connexion with the tinting of the ear lobes it is interesting to recall that it was in the rabbit's ear that Claude Bernard first demonstrated the action of the vasoconstrictor fibres, and it is now known that capillaries as well as arteries are influenced by vasoconstrictor stimulation. Stimulation of the vasoconstrictor centre in the brain would, by bringing about stasis in localized areas, induce there an increased venosity of the blood. This explains why the facies can be obvious while the person concerned remains symptomless.

As illustrative of the application of the vasomotor theory to the T.N.T. fac:es, I refer to an appeal in the British Medical Journal of November 15,1930 (p. 848) from the medical officer of a public school for diagnosis in the case of a boy who, after receiving dental treatment which left a fibre stopping in an incisor tooth, drew, while in the school laboratory, a few drops of aniline dye into his mouth from a pipette. He very soon 
collapsed and developed on the face, neck, and hands a " deep blue-black colour." The replies diagnosed the occurrence as a case of aniline poisoning with formation of methaemoglobinaemia. Commenting on the case, I drew attention (Journal, $1930,2,980)$ to the fact that there was not enough benzene chemical (nitro or amido) in a few drops of dye to cause methaemoglobinaemia, and suggested that the dye soaked into the fibre stopping and dissolved in the lipoids in the incisive nerve and thus reached the brain. The blueness and the distribution could not have been a blood change, but could arise from action of the sympathetic or central nervous system, or of both.

The works medical officer would do well to keep both theories in mind. In the symptomless cases the vasomotor view explains the shock-like pallor of the cheeks, the tinted ear lobes, and coloured lips, but some types of T.N.T. poisoning may develop blood changes, and a symptomless case may through continued exposure become one of a different and serious type.

\section{T.N.T. Absorption v. T.N.T. Poisoning}

Not every case showing the T.N.T. facies, even when associated with a positive Webster test, should be regarded as T.N.T. poisoning or be notified as such, otherwise many unnecessary notifications will be made and the position as regards the T.N.T. health hazard confused. The dividing line between absorption and poisoning will, of course, be drawn according to the experience of the medical officer and the opportunities he has for clinical investigation-for example, Webster test, etc. The correct practice in cases in which the facies is pronounced and Webster's test is positive is to suspend such workers from T.N.T. contact (not from work) for a week or ten days. If the same worker requires suspension several times he is tested for susceptibility (see Webster's test), and the intensity of a positive result in relation to the amount of exposure he has been subjected to after a previous negative result determines whether or not he is classified "susceptible" and removed permanently from contact with T.N.T.

Symptoms which may accompany the characteristic facies and still retain the case in the category of absorption are: onset of breathlessness, constriction felt beneath the midsternum, and malaise. Symptoms which point to systemic poisoning are: gastric ache felt in the early morning; anorexia which has been preceded by increased appetite ; physical and mental lassitude ; jaundice, exceedingly slight at onset, often first observed as a tinge in the sclerotic ; nausea and headache combined; and substernal constriction plus T.N.T. facies.

In some factories I have come across instances in which this substernal constriction was diagnosed as cardiac in origin and the worker certified to be suffering from heart strain. It is easy to make this mistake if the worker's doctor is unaware that the physical effort involved is most unlikely to cause heart strain, and also if the examination has taken place under bad or artificial lighting conditions. The symptom is more often met with in young women of the slightly anaemic type than in others, and the pain or ache complained of may be oesophageal in origin. When it is accompanied by a definitely positive Webster test the case is akin to that of gastric ache placed in the systemic poisoning category.

Doctors in munitions areas must not conclude from the scheme of medical supervision laid down as being essential that contact with T.N.T. necessarily creates sickness, for such is far from being the case. In a large munitions works in the North the manufacture of T.N.T. has gone on unceasingly for the past sixteen years without a single case of serious illness from T.N.T. contact, and many of the workers have been on the job all this time.
Webster's Test.-A description of Webster's test and its interpretation cannot (for reasons of space) be given here, but briefly it is a transient colour reaction indicating, when positive, that absorbed T.N.T. has been acted on during metabolism and has been excreted in altered form. The test in no way denotes systemic poisoning. Positive results can be graded from "trace" to "intense," and this standard helps greatly in the check-up on potential sickness and on susceptibles, since the intensity of a positive result can be considered in relation to the known exposure to T.N.T.

It cannot be too strongly urged on practitioners in munitions areas that they should make quick contact with the works medical officer when they are consulted by women who are exposed to T.N.T. and whose symptoms suggest an industrial origin. This attitude is particularly desirable when the altered health occurs within four months of employment.

\section{THE PROBLEM OF OPERATION FOR RETROVERTED UTERUS}

BY

CORALIE RENDLE-SHORT, M.B., Ch.B., M.R.C.O.G.

Tutor in Obstetrics, University of Bristol

Whether or not to advise operation in cases of backward displacement of the uterus has always been one of the most controversial subjects in gynaecology. The fact that there are so many different methods of correcting the retroverted uterus-some textbooks devote as many as eighty pages to describing different techniques-shows that there is no universally satisfactory method of operative treatment. However, although many writers either recommend or decry the operation, recent statistics of cases well followed up are not numerous. The out-patient departments of most gynaecological hospitals can produce several unfortunate women who have had some operation or other performed for backward displacement and are no better, and, perhaps what is even more important, the general practitioner who has persuaded some patient to have such an operation done, and finds her symptoms still continue, is puzzled as to what to advise for similar sufferers. In fact, he may get a general impression that all such operations are useless. The question remains, Is he justified in this opinion? It is a well-known fact that the grumbler always reappears: the cured and grateful patient often is not seen again.

The successful results of various operations for backward displacement can be divided into two classes: first, those which are an anatomical success, the uterus being in the anteverted position on examination ; and, secondly, those which are also cured of their symptoms-subjective success. It has been found that these two do not always go together.

\section{The Literature}

As usual, American writers report the largest number of cases. Graves and Smith (1931), examining 3,358 patients, found that after two years the anatomical cures were $75 \%$, symptomatic cures $63.4 \%$, and relieved $28.2 \%$. They used the Olshausen operation, in which the uterine ends of the round ligaments are stitched to the anterior abdominal wall. Hurd (1927) (1,000 cases in five and a half years, done by many different methods) found $88 \%$ successes in the whole series, both anatomical and symptomatic, with $12 \%$ failures. The purely anatomical successes were $96 \%$ for the whole series. He found that the Gilliam operation was the best for curing abdominal pain and backache but was not of very much value for sterility. Abdominal pain was the most frequent symptom remaining after operation. Gardner (1935) (145 cases), using a combined technique 